\title{
How to use ambulatory blood pressure monitoring in resistant hypertension
}

\author{
Elizabeth S Muxfeldt and Gil F Salles
}

\begin{abstract}
Resistant hypertension is defined as an uncontrolled office blood pressure (BP) despite the use of at least three antihypertensive drugs, in adequate doses and combinations, preferentially including one diuretic. It is a clinical diagnosis based on office BP measurements. Ambulatory BP monitoring (ABPM) is the cornerstone in the management of patients with resistant hypertension, as it is mandatory for diagnosis, treatment, follow-up and prognosis. In relation to diagnosis, ABPM measurements have classified patients with resistant hypertension into four subgroups: true, white-coat, controlled and masked resistant hypertension. This classification largely defines the therapeutic approach and the follow-up for each group. In this way, the target of antihypertensive treatment is ambulatory BP control and not office BP control. Chronotherapy based on ABPM values might frequently lead to a more rational treatment regimen. In relation to prognosis, uncontrolled ambulatory BP levels at baseline identify a subgroup of patients with a very high cardiovascular risk profile and a significantly worse prognosis. ABPM parameters can provide a better cardiovascular risk stratification than other traditional risk factors and office BPs.
\end{abstract} Hypertension Research (2013) 36, 385-389; doi:10.1038/hr.2013.17; published online 21 March 2013

Keywords: ambulatory blood pressure monitoring; resistant hypertension; treatment and prognosis

\section{INTRODUCTION}

Resistant hypertension is one of the well-established indications for ambulatory blood pressure (BP) monitoring (ABPM) performance, ${ }^{1-3}$ but in clinical practice its usefulness is wider than it seems to be. By definition, resistant hypertension is a condition of uncontrolled office BP in spite of using three or more antihypertensive medications. In addition, patients using four or more drugs with controlled office $\mathrm{BP}$ are also considered resistant. ${ }^{4}$ Although the definition is based on office BP measurements, ABPM is a very important tool not only for diagnosis, but also for treatment and follow-up, because of the high magnitude of the white-coat effect in these patients. ${ }^{5}$ Moreover, ambulatory BP and other ABPM parameters are important predictors of cardiovascular morbidity and mortality, ${ }^{6,7}$ whereas office $\mathrm{BP}$ seems to have a poor prognostic value. ${ }^{6}$ In the following sections, we will separately discuss the usefulness of ABPM for diagnosis, for treatment and follow-up, and for cardiovascular risk stratification and prognosis. In addition, we briefly discuss the potential role of home BP monitoring (HBPM) in resistant hypertension management. Finally, a full recommendation on how ABPM should be used in resistant hypertensive patient management will be provided.

\section{DIAGNOSIS}

Office BP is used to identify patients with resistant hypertension according to the definition, whereas ABPM classify those patients into four subgroups (Figure 1). If the office BP is controlled using four or more drugs, ${ }^{4}$ ABPM defines two groups: controlled resistant hypertension (office $\mathrm{BP}<140 / 90 \mathrm{~mm} \mathrm{Hg}$, and both daytime $\mathrm{BP}<135 / 85 \mathrm{~mm} \mathrm{Hg}$ and nighttime $\mathrm{BP}<120 / 70 \mathrm{~mm} \mathrm{Hg}$ ) and masked resistant hypertension (office $\mathrm{BP}<140 / 90 \mathrm{~mm} \mathrm{Hg}$, and either daytime $\mathrm{BP} \geqslant 135 / 85 \mathrm{~mm} \mathrm{Hg}$ or nighttime $\mathrm{BP} \geqslant 120$ / $70 \mathrm{~mm} \mathrm{Hg}$ ). In a large Japanese cohort, using HBPM, evaluating 528 resistant hypertensive patients, controlled resistant hypertension was found in $17.8 \%$ of patients and masked resistant hypertension in $23.5 \% .{ }^{8}$ There is no study analyzing large series of these two specific groups with ABPM. ${ }^{9}$ In our own cohort, after a median of 5 years of follow-up, of 473 resistant hypertensive patients, all of them with initially uncontrolled office BPs, 94 patients (20\%) achieved office BP control $(<140 / 90 \mathrm{~mm} \mathrm{Hg}$ at least on two consecutive visits). From these patients, $58(62 \%)$ had sustained controlled BP on a subsequent ABPM, whereas 36 (38\%) had masked resistant hypertension. On the other hand, from those 379 patients $(80 \%)$ who persisted with uncontrolled office BPs, 153 (40\%) had controlled ambulatory BPs (white-coat resistant hypertension) and the remaining 226 patients (60\%) had truly uncontrolled ambulatory BP levels (true resistant hypertension; unpublished observation).

Indeed, if the office BP still remains uncontrolled after initial therapeutic adjustments and adherence checking, the next step is also to submit resistant hypertensive patients to $24 \mathrm{~h}$ ABPM to identify whether BP is really uncontrolled, namely true resistant hypertension (office $\mathrm{BP} \geqslant 140 / 90 \mathrm{~mm} \mathrm{Hg}$, and either daytime $\mathrm{ABPM} \geqslant 135 / 85 \mathrm{~mm} \mathrm{Hg}$ 


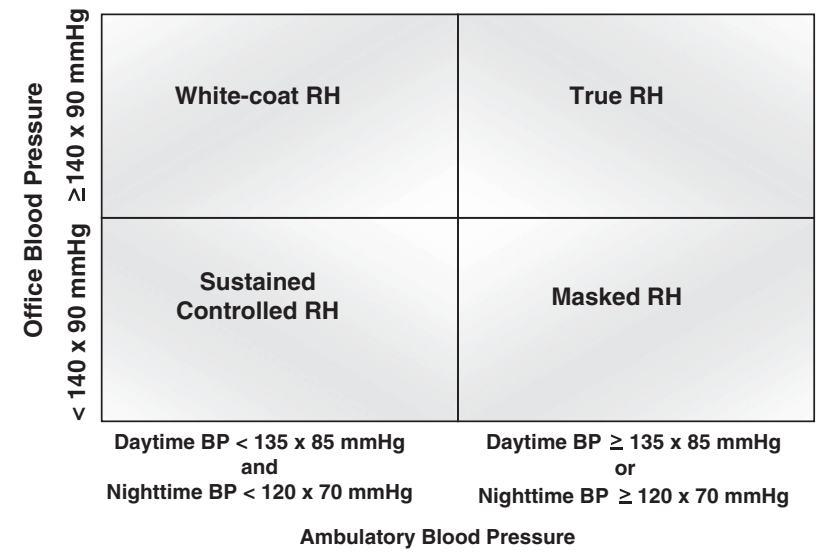

Figure 1 Classification of resistant hypertensive patients into four subgroups according to office and ambulatory blood pressure measurements: controlled, masked, white-coat and true resistant hypertension.

or nighttime $\mathrm{BP} \geqslant 120 / 70 \mathrm{~mm} \mathrm{Hg}$ ) or controlled, white-coat resistant hypertension (office $\mathrm{BP} \geqslant 140 / 90 \mathrm{~mm} \mathrm{Hg}$, and both daytime $\mathrm{ABPM}<135 / 85 \mathrm{~mm} \mathrm{Hg}$ and nighttime $\mathrm{BP}<120 / 70 \mathrm{~mm} \mathrm{Hg}){ }^{5}$ Whitecoat resistant hypertension was defined more than 10 years ago, ${ }^{10}$ and its prevalence in different series is as high as $40 \%$ of patients defined as resistant hypertensives. ${ }^{5,11-13}$ This classification, outlined in Figure 1, has important consequences, both for defining the therapeutic approach $^{4}$ and for prognosis. ${ }^{6}$

True resistant hypertension diagnosis is associated with a worse cardiovascular risk profile..$^{9,11-14}$ Younger age (1-13 $^{13}$ and male gender ${ }^{9,12,13}$ are more prevalent in this group of resistant hypertensive patients, as well as some cardiovascular risk factors, such as physical inactivity, ${ }^{12}$ diabetes and current smoking. ${ }^{9,13}$ They also presented a higher prevalence of subclinical organ damage, ${ }^{1}$ such as left ventricular hypertrophy $y^{5,9,12,13}$ and microalbuminuria, ${ }^{12,13}$ and also more prevalent cardiovascular diseases. ${ }^{12-14}$

In some series, office BPs are similar in the two groups, ${ }^{5,11}$ but in larger series ${ }^{9,12,13}$ true resistant hypertensive patients presented with higher office systolic BP (SBP) and diastolic BP (DBP). Moreover, the main important differences are found in the ABPM profile. ${ }^{5,10-14}$ Besides the expected differences in ambulatory BPs in the three periods (daytime, nighttime and $24 \mathrm{~h}$ ), ${ }^{5}$ a large systolic and diastolic white-coat effect is found in the two groups, reaching 50 and $24 \mathrm{~mm} \mathrm{Hg}$, respectively, in white-coat resistant hypertension, and 30 and $13 \mathrm{~mm} \mathrm{Hg}$, respectively, in true resistant hypertension, ${ }^{12,15}$ indicating the need to follow up all resistant hypertensive patients with ABPM. Comparing resistant hypertensive patients with controlled patients using three or less drugs, the white-coat effect is clearly higher in the former group. ${ }^{16}$

Once again, pointing to a higher cardiovascular risk in true resistant hypertension group, other parameters, such as a widened ambulatory pulse pressure ${ }^{5,12}$ and a higher prevalence of nondippers ${ }^{5}$ and risers, ${ }^{13}$ are also more frequently found in true resistant hypertensives than in white-coat resistant hypertensive patients, although the nocturnal BP reduction is similar in the majority of reported series. ${ }^{1-13}$

Using a simple score, it is roughly possible to predict true resistant hypertension without submitting patients to ABPM. ${ }^{12}$ This score includes male gender, office $\mathrm{SBP} \geqslant 180 \mathrm{~mm} \mathrm{Hg}$, serum potassium $<4.5 \mathrm{mmoll}^{-1}$, fasting glucose $>7.0 \mathrm{mmoll}^{-1}$, proteinuria $>200 \mathrm{mg}$ per $24 \mathrm{~h}$ (or albuminuria $\geqslant 30 \mathrm{mg}$ per $24 \mathrm{~h}$ ) and sexadjusted Cornell's voltage $>2.6 \mathrm{mV}$ (or echocardiographic left ventricular hypertrophy). Even though it presented a positive predictive value for true resistant hypertension of $90 \%$, the sensitivity was too low (32\%) and it is not possible to predict white-coat resistant hypertension. In this way, almost $40 \%$ of these subjects were misclassified. ${ }^{17}$

\section{FOLLOW-UP AND TREATMENT}

Treatment should be based on ABPM values, and the therapeutic target should be controlled BP in ABPM and not in office BP, because of the well-described high magnitude of white-coat effect in all resistant hypertensive patients. ${ }^{5,12,16,18}$ This approach will avoid overtreatment and, consequently, more drug adverse effects and lower adherence.

In this way, in patients with true resistant hypertension and masked resistant hypertension, for each therapeutic scheme adjustment, both in the dosage of currently using medications, as well as the addition of a new antihypertensive drug, a new ABPM is necessary to evaluate BP control. These patients should also be investigated for secondary causes of hypertension. ${ }^{4,19,20}$

Otherwise, in patients with a first diagnosis of white-coat resistant hypertension, beyond the maintenance of the therapeutic regimen, a confirmatory ABPM should be done in 3 months. If daytime SBP is $>115 \mathrm{~mm} \mathrm{Hg}$, ABPM should be repeated in 6 months, because of a higher chance of becoming true resistant hypertension. In patients with daytime $\mathrm{SBP} \leq 115 \mathrm{~mm} \mathrm{Hg}$, ABPM can be repeated yearly. ${ }^{21}$ In patients with sustained controlled resistant hypertension, the therapeutic scheme should be maintained, but there is no definition in literature about the adequate time interval to repeat $A B P M$, although a yearly examination seems reasonable to ascertain the persistence of ambulatory BP control.

Another important use of ABPM in resistant hypertension is the adjustment of therapeutic scheme by chronotherapy, ${ }^{22}$ particularly regarding the nighttime period. A previous report ${ }^{23}$ evaluating 700 resistant hypertensive patients submitted to $48 \mathrm{~h} \mathrm{ABPM}$, in which 401 patients were using at least 1 drug at bedtime and 299 were in single morning doses; the former group presented $20.7 \%$ of controlled ambulatory BP against $13 \%$ of the latter one, and also a lower prevalence of nondipping pattern $(56.9 \%$ vs. $81.9 \%)$.

In another study, ${ }^{24}$ a total of 250 resistant hypertensive patients were divided again into two groups. The first one used all three drugs in a single morning dose and the other used two drugs at morning and one drug at bedtime. After 12 weeks of treatment, the second group decreased ambulatory SBPs in the three periods: diurnal (average reduction $4.1 \pm 8.6 \mathrm{~mm} \mathrm{Hg}$ ), nocturnal $(10.9 \pm 9.4 \mathrm{~mm} \mathrm{Hg}$ ) and $24 \mathrm{~h}(6.4 \pm 8.1 \mathrm{~mm} \mathrm{Hg})$ versus the first group, with nonsignificant changes of $-0.3 \pm 8.4 \mathrm{~mm} \mathrm{Hg}$ (diurnal), $\quad-1.3 \pm 8.8 \mathrm{~mm} \mathrm{Hg}$ (nocturnal) and $-0.5 \pm 7.8 \mathrm{~mm} \mathrm{Hg}(24 \mathrm{~h})$. Moreover, the group of single morning dose showed a twofold higher prevalence of the nondipping pattern $(86.4 \%$ vs. $43.2 \%)$ and a lower nocturnal fall both in SBP $(2.1 \pm 9.1 \%$ vs. $9.4 \pm 8.5 \%)$ and in $\mathrm{DBP}(7.7 \pm 8.4 \%$ vs. $14.9 \pm 8.5 \%)$ in comparison with those with bedtime medication.

In a large series of 1794 resistant hypertensive patients, controlled resistant hypertension was more prevalent in patients using bedtime medication. ${ }^{25}$ Among the 1306 true resistant hypertensive patients, those using at least one drug at bedtime presented lower mean 24-h SBPs and lower prevalence of the nondipping pattern. They also showed a better metabolic profile, with lower values of fasting glycemia, total cholesterol, low-density lipoprotein-cholesterol and microalbuminuria. ${ }^{25}$ This important change in the profile of ABPM in resistant hypertensive patients using at least one drug at bedtime could probably improve the prognosis of these patients, not only 
because of a better controlled 24-h ambulatory $\mathrm{BP},{ }^{6,9}$ but also because of a decrease in nighttime BPs and higher prevalence of the normal dipping pattern, which are related to less subclinical organ damage, such as microalbuminuria, ${ }^{26,27}$ left ventricular hypertrophy ${ }^{26}$ and arterial stiffness. $^{28}$ A suitable nocturnal BP profile is actually associated with lower prevalence of overall target-organ damage ${ }^{26}$ and lower cardiovascular morbidity and mortality in resistant hypertension. ${ }^{6,7}$

\section{PROGNOSIS}

Although many studies demonstrated strong associations between the diagnosis of true resistant hypertension ${ }^{5,11-14}$ and other ABPMderived parameters with a worse cardiovascular risk profile, ${ }^{12,13,26-28}$ there are indeed few prospective studies evaluating the prognostic impact of office and ambulatory BP for cardiovascular morbidity and mortality in resistant hypertensive patients. ${ }^{6,7,9,15,29,30}$

The first one, published more than a decade ago, ${ }^{15}$ enrolled 86 resistant hypertensive patients with DBPs higher than $100 \mathrm{~mm} \mathrm{Hg}$, with a median follow-up of 49 months. Patients were divided into tertiles of mean 24-h DBP: lower than $88 \mathrm{~mm} \mathrm{Hg}$, between 88 and $97 \mathrm{~mm} \mathrm{Hg}$ and higher than $97 \mathrm{~mm} \mathrm{Hg}$. A total of 21 cardiovascular events occurred during the follow-up and the crude incidence was higher in the highest tertile subgroup (13.6 per 100 patient-years) than in the middle (9.5 per 100 patient-years) and in the lowest tertile subgroup (2.2 per 100 patient-years). A DBP higher than $97 \mathrm{~mm} \mathrm{Hg}$ was also an independent risk factor for future cardiovascular event occurrence in multivariate analysis, with a relative risk (RR) of 6.20 (95\% confidence interval $(\mathrm{CI}): 1.38-28.1 ; P<0.02)$ in relation to the reference subgroup with DBPs lower than $88 \mathrm{~mm} \mathrm{Hg}$.

In another study, ${ }^{9} 742$ general treated hypertensive patients, 466 patients with controlled office BPs (340 responder hypertensives and 126 with masked hypertension on ABPM) and 276 patients with resistant hypertension (146 white-coat resistant hypertension and 130 true resistant hypertension), were followed-up for a mean of $5.0 \pm 2.9$ years. A total of 63 cardiovascular events occurred (11 fatal events) and the crude incidence rate per 100 patient-years was 0.87 (95\% CI: $0.50-1.40)$ for responder hypertensives, 2.42 (95\% CI: 1.36-4.00) for masked hypertensives, 1.20 (95\% CI: 0.52-2.36) for white-coat resistant hypertensives and 4.10 (95\% CI: 2.63-6.10) for true resistant hypertensive patients. In Cox regression analysis, comparing with responder subgroup, the baseline diagnosis of masked hypertension (RR: 2.28; 95\% CI: 1.10-4.70; $P<0.05$ ) and of true resistant hypertension (RR: $2.94 ; 95 \%$ CI: $1.02-8.41 ; P<0.05$ ) were important predictors for cardiovascular morbidity and mortality, whereas the white-coat resistant hypertension diagnosis implied no significant additional risk (RR: 1.22; 95\% CI: 0.45-3.34, $P=0.70)$. Age, smoking, low-density lipoprotein-cholesterol, diabetes and left ventricular hypertrophy were also independent predictors of events, but true resistant hypertension at baseline was the strongest one.

Finally, from our own cohort of 556 resistant hypertensive patients followed up for a median of 4.8 years (maximum 9 years), three reports evaluated the prognostic importance of mean office and ambulatory BPs, ${ }^{6}$ nocturnal BP reductions and dipping patterns, ${ }^{7}$ and the ambulatory arterial stiffness index (AASI). ${ }^{29}$ The primary endpoints were a composite of fatal and nonfatal cardiovascular events, all-cause mortality and cardiovascular mortality. The secondary endpoints were isolated coronary events and strokes. Fatal and nonfatal cardiovascular events occurred in 109 patients and the crude event rate was 4.32 per 100 patient-years. There were 70 all-cause deaths, of which 46 were from cardiovascular causes.
Higher nighttime SBP (RR: 1.38; 95\% CI: 1.13-1.68; $P<0.01$ ) and DBP (RR: 1.36; 95\% CI: 1.10-1.69; $P<0.01$ ), both estimated for increments of $1-$ s.d., were the strongest predictors of cardiovascular morbidity and mortality, but all ambulatory BPs (24h, daytime and nighttime) predicted these events after full statistical adjustments (for age, sex, body mass index, diabetes mellitus, smoking, physical inactivity, dyslipidemia, previous cardiovascular diseases, serum creatinine level and number of antihypertensive drugs in use). A widened 24-h (RR: 1.22; 95\% CI: 1.00-1.48; $P<0.05$ ) and nighttime pulse pressure (RR: $1.27 ; 95 \% \mathrm{CI}: 1.04-1.55 ; P<0.05$ ) were weaker predictors of the composite endpoint, in relation to mean SBPs and DBPs. Surprisingly, no office BP showed any prognostic value. ${ }^{6}$ Moreover, the diagnosis of true resistant hypertension at baseline doubled the risk for fatal and nonfatal cardiovascular events (RR: 2.11; 95\% CI: $1.34-3.34 ; P<0.001$ ) and for all-cause mortality (RR: 2.00 ; 95\% CI: $1.12-3.55$; $P<0.05$ ) after full adjustments, in comparison with the white-coat resistant hypertension subgroup. ${ }^{6}$

The second report, ${ }^{7}$ focusing on the nocturnal BP reductions and the different dipping patterns as independent prognostic markers over and beyond other cardiovascular risk factors, including mean ambulatory BP levels, showed that the nondipping status was the predictor of the composite endpoint (RR: 1.74; 95\% CI: 1.12-2.71; $P<0.05$ ) and of cardiovascular mortality (RR: 2.31 ; 95\% CI: 1.09 4.92; $P<0.05$ ), but not of all-cause mortality. This effect was observed both in the nondipper and riser subgroups, whereas the extreme dippers had no unfavorable prognosis.

Regarding secondary endpoints, mean ambulatory BPs and, particularly, the true resistant hypertension diagnosis were mainly predictors of stroke occurrence (for true resistant hypertension, RR: 3.20; 95\% CI: $1.41-7.25, P<0.01),{ }^{6}$ whereas the nondipping pattern was an important risk marker mainly for coronary events (RR: 2.05; 95\% CI: $1.00-4.20 ; P<0.05){ }^{7}$

Finally, the third report ${ }^{29}$ evaluated the prognostic value of the AASI, a new index derived from ABPM recording and considered to be an indirect marker of arterial stiffness. After all adjustments, including ambulatory BPs and the nocturnal BP reduction patterns, the 24-h AASI was the most important risk marker for the composite endpoint (RR: 1.46; 95\% CI: 1.12-1.92; $P<0.01$; for increments of 1 -s.d.). Moreover, the strongest predictor of cardiovascular mortality was the nighttime AASI (1.73; 95\% CI: $1.13-2.65 ; P<0.05)$, whereas no AASI parameter predicted all-cause mortality.

In summary, at least four ABPM-derived parameters-the mean SBPs and DBPs, the diagnosis of true resistant hypertension (and possibly also of masked resistant hypertension), the presence of a nondipping pattern and a higher AASI-are cardiovascular risk markers in patients with resistant hypertension. ${ }^{30}$ It is at present unclear whether controlling these parameters will favorably affect the prognosis, although one study ${ }^{31}$ has suggested that decreasing nighttime SBP and reverting the nondipping patternby bedtime antihypertensive treatment may improve cardiovascular prognosis in general hypertensive patients.

\section{HBPM IN RESISTANT HYPERTENSION}

Although there is little evidence supporting the use of HBPM in patients with resistant hypertension, it may be useful, especially in situations when ABPM is not available. Moreover, its lower cost and higher patient acceptance might justify its use in this group of patients in whom ABPM is mandatory for diagnosis and follow-up.

Indeed, there are only two studies comparing ABPM and HBPM in resistant hypertensive patients, and both concluded that HBPM can 


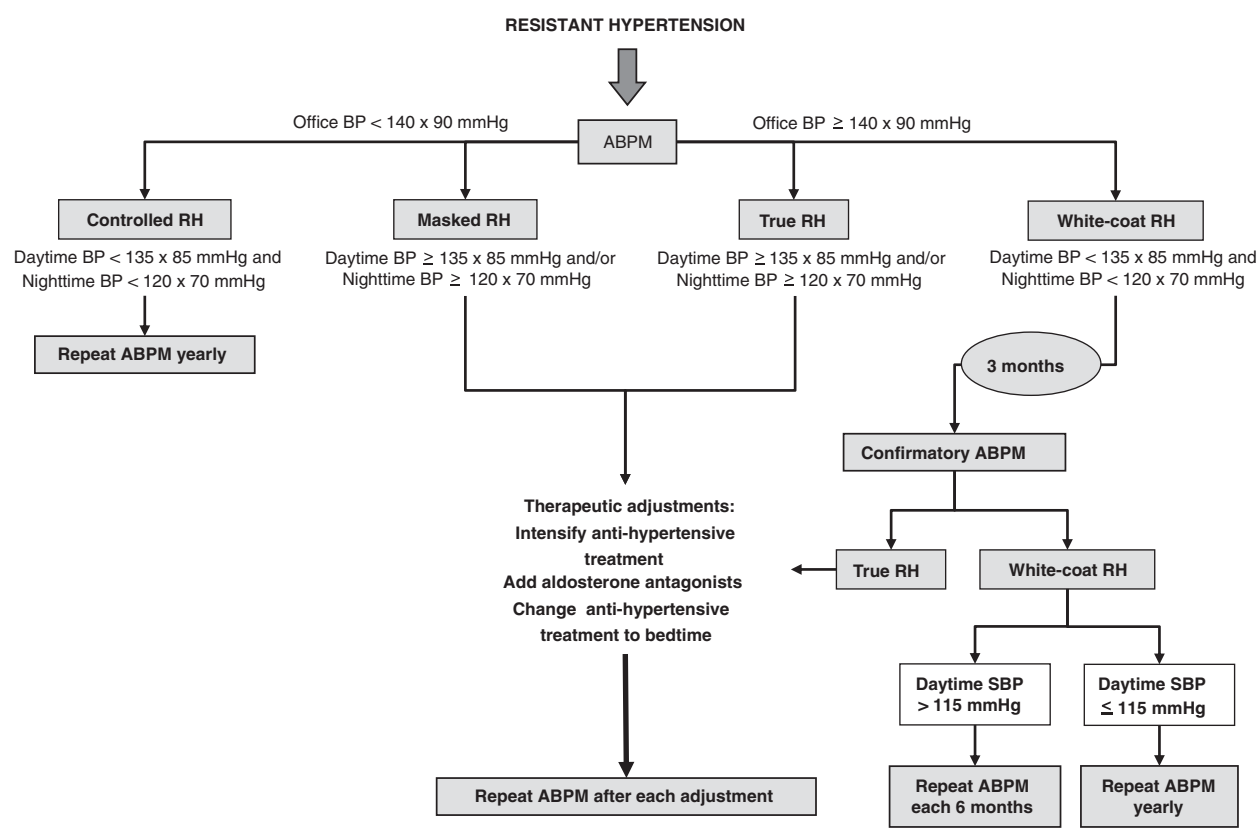

Figure 2 Recommended algorithm for ambulatory blood pressure monitoring use in patients with resistant hypertension.

be an alternative method for classifying true or white-coat resistant hypertension. ${ }^{32,33}$ However, in the first study only a moderate agreement between ABPM and HBPM was found in $86 \%$ of whitecoat resistant hypertensive patients $(\kappa=0.59)$ and $74 \%$ of true resistant hypertensive patients $(\kappa=0.46)$. The specificity, sensitivity, positive and negative predictive value of HBPM were 93\%, 63\%, 81\% and $83 \%$, respectively, for detecting white-coat resistant hypertension, and these values were $90 \%, 55 \%, 71 \%$ and $82 \%$, respectively, for detecting true resistant hypertension. ${ }^{32}$ In the second study, good correlations between ambulatory and home SBPs $(r=0.70)$ and DBPs $(r=0.69)$ were shown, ${ }^{33}$ although these correlations did not signify agreement between the two measurements. As mentioned previously, a Japanese population-based study (J-HOME) ${ }^{8}$ used HBPM to classify resistant hypertensive patients into true $(42.6 \%)$, white-coat $(16.1 \%)$, controlled $(17.8 \%)$ and masked resistant hypertension (23.5\%) subgroups.

One of the most important limitations of HBPM is the lack of nighttime sleep measurements. This is particularly relevant in resistant hypertension because of the well-demonstrated higher prognostic importance of the nocturnal BP profile than daytime BP in this group of patients, ${ }^{6,7,26-28}$ emphasizing the importance of the nocturnal BP control. ${ }^{24,31}$ Therefore, HBPM may be useful in resistant hypertension management for checking the response to each therapeutic adjustment prescribed for true and masked resistant hypertensive patients (if these interventions take into account daytime BP levels), and possibly also for ascertaining the persistence of BP control in patients initially diagnosed as white-coat or controlled resistant hypertension. ${ }^{21}$

\section{HOW TO USE ABPM IN RESISTANT HYPERTENSION}

$A B P M$ is mandatory for diagnosis, treatment, follow-up and prognosis of patients with resistant hypertension. Besides the correct classification in the four established subgroups-true resistant hypertension, white-coat resistant hypertension, controlled resistant hypertension and masked resistant hypertension-the current use of ABPM in those patients allows to achieve adequate BP control, avoiding overtreatment and, consequently, reducing adverse effects of drugs and increasing adherence to treatment. Moreover, ABPM is also an important tool to define prognosis, improving risk stratification in this group of patients with a high cardiovascular morbidity and mortality. Figure 2 presents a general scheme for ABPM use in resistant hypertensive patients. In patients with controlled ambulatory BP levels (sustained controlled resistant hypertension and white-coat resistant hypertension), ABPM shall be repeated at 6-12 months interval to assure the persistence of ambulatory BP control, irrespective of office BP levels. On the other hand, in patients with uncontrolled ambulatory BP levels (true resistant hypertension and masked resistant hypertension), ABPM shall be repeated whenever antihypertensive treatment is intensified, also irrespective of office BP changes. In this regard, aldosterone antagonists ${ }^{34,35}$ and bedtime antihypertensive treatment ${ }^{24,31}$ seem to have important roles, respectively, in controlling ambulatory BPs and in reverting abnormal dipping patterns. The overall antihypertensive treatment target in patients with resistant hypertension shall be keeping control of daytime and nighttime BPs. In general, these recommendations regarding $A B P M$ use in resistant hypertension mean that all resistant hypertensive patients will perform an ABPM at least yearly (or more frequently in selected cases of therapeutic interventions). In our own experience, this is feasible in clinical practice, although in some situations HBPM may be considered an alternative to frequent performance of ABPMs, particularly when sleep BP control is not in question.

\section{CONFLICT OF INTEREST}

The authors declare no conflict of interest.

\section{ACKNOWLEDGEMENTS}

This study was partially supported by grants from the Conselho Nacional de Desenvolvimento Científico e Tecnológico $(\mathrm{CNPq})$ and from the Fundação Carlos Chagas Filho de Amparo a Pesquisa do Estado do Rio de Janeiro (FAPERJ). 
1 Mancia G, de Backer G, Dominiczak A, Cifkova R, Fagard R, Germano G, Grassi G, Heagerty AM, Kjeldsen E, Laurent S, Narkiewicz K, Ruilope L, Rynklewicz A, Schmieder RE, Boudier HAJS, Zanchetti A. 2007 Guidelines for the Management of Arterial Hypertension. The Task force for the management of arterial hypertension of the European Society of Hypertension (ESH) and of the European Society of Cardiology (ESC). J Hypertens 2007; 25: 1105-1187.

2 Chobanian AV, Bakris GL, Black HR, Cushman WC, Green LA, Izzo JL, Jones DW, Materson BJ, Oparil S, Wright JT, Roccella EJ, the National High Blood Pressure Education Program Coordinating Committee. Seventh report of the Joint National Committee on Prevention, Detection, Evaluation and Treatment of High Blood Pressure. Hypertension 2003; 42: 1206-1252.

3 O'Brien E, Asmar R, Beilin L, Imai Y, Mancia G, Mengden T, Myers M, Padfield P, Palatini P, Parati G, Pickering T, Redon J, Staessen J, Stergiou G, Verdecchia P. Practice guidelines of the European Society of Hypertension for clinic, ambulatory and self blood pressure measurement. J Hypertens 2005; 23: 697-701.

4 Calhoun DA, Jones D, Textor S, Goff DC, Murphy TP, Toto RD, White A, Cushman WC, White W, Sica D, Ferdinand K, Giles TD, Falkner B, Carey RM. Resistant hypertension: diagnosis, evaluation, and treatment. A scientific statement from the American Heart Association Professional Education Committee of the Council for High Blood Pressure Research. Hypertension 2008; 51: 1403-1419.

5 Muxfeldt ES, Bloch KV, Nogueira AR, Salles GF. Twenty-four hour ambulatory blood pressure monitoring pattern of resistant hypertension. Blood Press Monit 2003; 8: 181-185.

6 Salles GF, Cardoso CRL, Muxfeldt ES. Prognostic influence of office and ambulatory blood pressures in resistant hypertension. Arch Int Med 2008; 168: 2340-2346.

7 Muxfeldt ES, Cardoso CRL, Salles GF. Prognostic value of nocturnal blood pressure reduction in resistant hypertension. Arch Int Med 2009; 169: 874-880.

8 Oikawa T, Obara T, Ohkubo T, Kikuya M, Asayama K, Metoki H, Komai R, Muria K, Hashimoto J, Totsune K, Imai Y, J-Home study group. Characteristics of resistant hypertension determined by self-measured blood pressure at home and office blood pressure measurements: the J-HOME study. J Hypertens 2006; 24: 1737-1743.

9 Pierdomenico SD, Lapenna D, Bucci A, Di Tommaso R, Di Mascio R, Manente BM, Caldarella MP, Neri M, Cuccurullo F, Mezzeti A. Cardiovascular outcome in treated hypertensive patients with responder, masked, false resistant, and true resistant hypertension. Am J Hypertens 2005; 18: 1422-1428.

10 Mezzetti A, Pierdomenico SD, Costantini F, Romano F, Bucci A, Di Gioacchino M, Cuccurullo F. White-coat resistant hypertension. Am J Hypertens 1997; 10: 1302-1307.

11 Veglio F, Rabbia F, Riva P, Martini G, Genova GC, Milan A, Paglieri C, Carra R, Chiandussi L. Ambulatory blood pressure monitoring and clinical characteristics of the true and white-coat resistant hypertension. Clin Exp Hypertens 2001; 23: 203-211.

12 Muxfeldt ES, Bloch KV, Nogueira AR, Salles GF. True resistant hypertension: is it possible to be recognized in the office? Am J Hypertens 2005; 18: 1534-1540.

13 de la Sierra A, Segura J, Banegas JR, Gorostidi M, de la Cruz JJ, Armario P, Oliveras A, Ruilope LM. Clinical features of 8295 patients with resistant hypertension classified on the basis of ambulatory blood pressure monitoring. Hypertension 2011; 57: 898-902.

14 Hernandez-del Rey R, Armario P, Martin-Baranera M, Sanchez P, Cardenas G, Pardell $\mathrm{H}$. Target-organ damage and cardiovascular risk profile in resistant hypertension. Influence of the white-coat effect. Blood Press Monit 1998; 3: 331-337.

15 Redon J, Campos C, Narciso ML, Rodicio JL, Pascual JM, Ruilope LM. Prognostic value of ambulatory blood pressure monitoring in refractory hypertension: a prospective study. Hypertension 1998; 31: 712-718.

16 de la Sierra A, Banegas JR, Oliveras A, Gorostidi M, Segura J, de la Cruz JJ, Armario P, Ruilope LM. Clinical differences between resistant hypertensives and patients treated and controlled with three or less drugs. Hypertension 2012; 30: 1211-1216.
17 Redon J. Managing resistant hypertension: not just a question of numbers. Am J Hypertens 2005; 18: 1541-1542.

18 Syrseloudis D, Andrikou I, Andrikou E, Dimitriadis K, Stefanadis C. Ambulatory blood pressure monitoring in resistant hypertension. Int J Hypertension 2011; 2011: 285612.

19 Oliveras A, de la Sierra A. New developments in the diagnosis and management of resistant hypertension. Curr Med Chem 2012; 19: 1210-1218.

20 Pedrosa RP, Drager LF, Gonzaga CC, Sousa MG, de Paula LK, Amaro AC, Bortolotto LA, Krieger EM, Bradley TD, Lorenzi Filho G. Obstructive sleep apnea: the most common secondary cause of hypertension associated with resistant hypertension. Hypertension 2011; 58: 811-817.

21 Muxfeldt ES, Fiszman R, de Souza F, Viegas B, Oliveira FC, Salles GF. Appropriate time interval to repeat ambulatory blood pressure monitoring in patients with white-coat resistant hypertension. Hypertension 2012; 59: 384-389.

22 Hermida RC, Ayala DE, Portaluppi F. Circadian variation of blood pressure: the basis for the chronotherapy of hypertension. Adv Drug Deliv Rev 2007; 59: 904-922.

23 Hermida RC, Ayala DE, Calvo C, López JE, Mojón A, Fontao MJ, Soler R, Fernández JR. Effects of time of day of treatment on ambulatory blood pressure pattern of patients with resistant hypertension. Hypertension 2005; 46: 1053-1059.

24 Hermida RC, Ayala DE, Fernández JR, Calvo C. Chronotherapy improves blood pressure control and reverts the nondipper pattern in patients with resistant hypertension. Hypertension 2008; 51: 69-76.

25 Hermida RC, Ayala DE, Mojon A, Fernández JR. Effects of time of antihypertensive treatment on ambulatory blood pressure and clinical characteristics of subjects with resistant hypertension. Am J Hypertens 2010; 23: 432-439.

26 Muxfeldt ES, Salles GF. Pulse pressure or dipping pattern: which one is a better cardiovascular risk marker in resistant hypertension? J Hypertens 2008; 26: 878-884.

27 Oliveras A, Armario P, Martell N, Ruilope LM, de la Sierra A. Urinary albumin excretion is associated with nocturnal systolic blood pressure in resistant hypertensives. Hypertension 2011; 57: 556-560.

28 Castelpoggi $\mathrm{CH}$, Pereira VS, Fiszman R, Cardoso CRL, Muxfeldt ES, Salles GF. A blunted decrease in nocturnal blood pressure is independently associated with increased aortic stiffness in patients with resistant hypertension. Hypertens Res 2009; 32: 591-596.

29 Muxfeldt ES, Cardoso CR, Dias VB, Nascimento AC, Salles GF. Prognostic impact of the ambulatory arterial stiffness index in resistant hypertension. J Hypertens 2010; 28: 1547-1553.

30 de Souza F, Muxfeldt ES, Salles GF. Prognostic factors in resistant hypertension: implications for cardiovascular risk stratification and therapeutic management. Exp Rev Cardiovasc Ther 2012; 10: 735-745.

31 Hermida RC, Ayala DE, Mojón A, Fernández JR. Decreasing sleep-time blood pressure determined by ambulatory monitoring reduces cardiovascular risk. J Am Coll Cardiol 2011; 58: 1165-1173.

32 Nasothimiou EG, Tzamouranis D, Roussias LG, Stergiou GS. Home versus ambulatory blood pressure monitoring in the diagnosis of clinic resistant and true resistant hypertension. J Hum Hypertens 2011; 26: 696-700.

33 Marui FR, Bombig MT, Francisco YA, Thalenberg JM, Fonseca FA, Souza D, Costa Fde A, Izar MC, Carvalho AC, Póvoa R. Assessment of resistant hypertension with home blood pressure monitoring. Arq Bras Cardiol 2010; 95: 536-540.

34 Chapman N, Dobson J, Wilson S, Dahlof B, Sever PS, Wedel H, Poulter NR. Effect of spironolactone on blood pressure in subjects with resistant hypertension. Hypertension 2007; 49: 839-845.

35 de Souza F, Muxfeldt E, Fiszman R, Salles G. Efficacy of spironolactone therapy in patients with true resistant hypertension. Hypertension 2010; 55: 147-152. 